\title{
Cáncer gástrico: una enfermedad prevenible. Estrategias para intervención en la historia natural
}

\author{
Gastric cancer is a preventable disease: Strategies for intervention in its \\ natural history
}

Ricardo Oliveros, ${ }^{1 *}$ Raúl E. Pinilla, ${ }^{1}$ Helena Facundo Navia, ${ }^{1}$ Ricardo Sánchez Pedraza. ${ }^{2}$

1 Especialista en Cirugía General, Gastroenterología y Endoscopia Digestiva. Instituto Nacional de Cancerología. Bogotá D. C., Colombia.

2 Médico Cirujano Especialista en Psiquiatría Magíster en Epidemiología Clínica y Especialista en Estadística. Universidad Nacional de Colombia e Instituto Nacional de Cancerología. Bogotá D. C., Colombia.

*Correspondencia: roliveros4@yahoo.es.

Fecha recibido: $22 / 06 / 18$

Fecha aceptado: 19/11/18

\begin{abstract}
Resumen
El cáncer gástrico es un problema de salud pública. Las cifras de mortalidad y supervivencia son impresentables en nuestro país. En Colombia no existe ningún programa ni estrategias de diagnóstico temprano, ni tampoco es priorizado como un problema de salud. Los trabajos existentes demuestran que la mayoría de los pacientes cuando son diagnosticados presentan estadios avanzados.

Un $90 \%$ de los canceres gástricos se consideran consecuencia de un largo proceso inflamatorio sobre la mucosa gástrica. La infección por Helicobacter pylori es la principal etiología de la gastritis, la cual puede progresar a atrofia, metaplasia, displasia y cáncer. La atrofia gástrica establece un campo de cancerización más propenso a cambios moleculares y fenotípicos que terminan en un cáncer en crecimiento. Es claro que la historia natural proporciona un racional entendimiento clínico patológico para estrategias de prevención primaria y secundaria. Una evidencia bien establecida demuestra que la combinación de las estrategias primarias (erradicación del $H$. pylori) y secundarias (diagnóstico y seguimiento endoscópico de lesiones premalignas) pueden prevenir o limitar la progresión de la carcinogénesis gástrica. El riesgo de cáncer gástrico asociado con la gastritis por $H$. pylori puede ser estratificado de acuerdo con la gravedad y extensión de la atrofia de la mucosa gástrica. Esta aproximación está adaptada a diferentes países, de acuerdo con su incidencia específica de cáncer gástrico, condición socioeconómica y factores culturales, que requiere de la participación complementaria de los gastroenterólogos, los cirujanos, los oncólogos y patólogos.

Frente a este problema de salud pública no hay ninguna acción por parte de las autoridades de salud ni del gremio médico. Por tanto, se revisan las estrategias de manejo que permitan intervenir la historia natural de la enfermedad con el objetivo de disminuir la incidencia y mortalidad.

La implementación y estandarización de estas estrategias de manejo en nuestro medio podrán beneficiar a los pacientes con riesgo incrementado para cáncer gástrico y pueden implementarse (en países sin programas de tamizaje) de una forma racional, similar a como se está haciendo con el cáncer colorrectal, en todo el mundo.
\end{abstract}

\section{Palabras clave}

Cáncer gástrico, Helicobacter pylori, prevención, historia natural.

\section{INTRODUCCIÓN}

El cáncer gástrico (CG) es un problema de salud pública a nivel mundial, a pesar de la disminución en su incidencia y mortalidad en los últimos años (1). De acuerdo con GLOBOCAN, 1033701 nuevos casos por CG se presentaron en 2018 y más de 782685 muertes por esta enfermedad ocurrieron durante el mismo año (2). El CG en el mundo representa el 5,7 \% de todos los nuevos casos de cáncer y el 8,2 \% del total de muertes por cáncer $(2,3)$. Japón y Corea tienen las cifras más altas en incidencia en el mundo. Son áreas de alta incidencia: Asia, Europa Oriental, América del Sur y América Central; y son áreas de baja incidencia: el sur del Asia, norte y este de África, Norteamérica, Australia y Nueva Zelandia (4). Japón y Corea tiene las cifras más altas en incidencia en el mundo. En Japón, donde el CG permanece como el tipo de cáncer más común 


\begin{abstract}
Gastric cancer is a public health problem, but there are no usable mortality and survival statistics for Colombia. The country has no early diagnosis program or strategy, and gastric cancer is not prioritized as a health problem. Existing studies show that most patients are in advanced stages by the time they are diagnosed.

Ninety percent of gastric cancers are considered to be consequences of long inflammatory processes in the gastric mucosa. H Pylori infections are the most common etiology of gastritis which can progress to atrophy, metaplasia, dysplasia and cancer. Gastric atrophy establishes a cancerization field which is prone to molecular and phenotypic changes that end in cancerous growth. It is well understood that a disease's natural history provides a rational pathological clinical understanding for primary and secondary prevention strategies. Well-established evidence shows that the combination of primary ( $\mathrm{H}$ pylori eradication) and secondary strategies (diagnosis and endoscopic follow-up of pre-malignant lesions) can prevent or limit the progression of gastric carcinogenesis. The risk of gastric cancer associated with $\mathrm{H}$ pylori gastritis can be stratified according to the severity and extent of atrophy of the gastric mucosa. This approach has been adapted to many different countries according to specific incidences of gastric cancer, socio-economic conditions and cultural factors. This requires the complementary participation of gastroenterologists, surgeons, oncologists and pathologists. In the face of this public health problem, there has been no action by health authorities or the medical association. For this reason, we have reviewed management strategies that allow intervening into the natural history of the disease to reduce its incidence and mortality rate.

The implementation and standardization of these management strategies in our environment may benefit patients who are at high risk for gastric cancer. These strategies can be implemented in a rational way, similar to what is being done with rectal cancer, in countries without screening programs all over the world.
\end{abstract}

\title{
Keywords
}

Gastric cancer, helicobacter pylori, prevention, natural history.

tanto en hombres como en mujeres, las cifras de incidencia son de 69,2/100 000 habitantes y de 28,6/100 000 habitantes, respectivamente (4).

En Colombia, según GLOBOCAN, en 2018 se detectaron 7419 casos nuevos de CG (7,3\%), de los cuales murieron 5505. El CG ocupó en incidencia para ese año el tercer lugar, luego del cáncer de mama y próstata, seguido por el cáncer de pulmón y colorrectal. Para el 2018 el CG representó la primera causa de mortalidad por cáncer $(13,7 \%)$ (5).

El riesgo de desarrollar CG se incrementa con la edad, ocurre más frecuentemente entre los 50 y 80 años de edad $y$ es infrecuente en personas menores de 30 años. (6).

A pesar de la importante carga de enfermedad, en Colombia no existe ningún programa de control y prevención de CG, ni tampoco es priorizado como un problema de salud pública. Los trabajos de investigación existentes demuestran que la mayoría de los pacientes con CG presenta estadios avanzados cuando se diagnostica y esto se traduce en cifras muy bajas de supervivencia (7).

El CG es multifactorial, con una compleja interacción de agentes infecciosos (Helicobacter pylori y virus de EpsteinBarr), medioambientales (alta ingesta de sal, consumo de tabaco y dietas pobres en fibra, frutas y verduras) y con componente genético (antecedentes familiares con CG). El agente causal más importante es el $H$. pylori, una bacteria cuya infección se adquiere en la infancia y, en ausencia de un tratamiento adecuado, puede persistir toda la vida; esto induce una respuesta inflamatoria crónica que condiciona de manera variable al desarrollo de atrofia, metaplasia, displasia y, por último, CG (8).

La prevención primaria del CG está dirigida a una dieta adecuada, rica en fibra, con mayor ingesta de frutas y verduras; y a un diagnóstico y tratamiento de la infección por $H$. pylori tempranamente en la vida, estrategia que debe realizarse antes de que existan cambios en la mucosa gástrica de atrofia y metaplasia intestinal. La prevención secundaria está dirigida al diagnóstico y seguimiento de las lesiones preneoplásicas como la atrofia y la metaplasia intestinal, fundamentadas en una escala de gravedad de acuerdo con la estadificación histológica conocida como el sistema Operative Link on Gastritis Assessment (OLGA) y Operative Link on Gastritis/Intestinal-Metaplasia Assessment (OLGIM) (9).

Estas últimas recomendaciones se establecen porque los programas de tamización no son posibles en países con economías bajas o intermedias, como en Colombia, donde los recursos deben estar dirigidos a problemas inmediatos considerados de mayor urgencia (9).

Infortunadamente, en Colombia el diagnóstico se realiza en estadios avanzados, cuando no existe la posibilidad de curación para esta enfermedad. Frente a este problema de salud pública no hay ninguna acción por parte de las autoridades de salud ni del gremio médico; por tanto, debe ser un objetivo proponer a la comunidad médica del país estrategias de trabajo sobre la historia natural que permitan disminuir su incidencia y mortalidad (9). 
El CG es una enfermedad prevenible. Existen estrategias dentro de la literatura con adecuados niveles de evidencia que permiten actuar dentro de la historia natural con el objetivo de disminuir su incidencia y las cifras de mortalidad, y mejorar la supervivencia a través de diagnósticos más tempranos. La implementación de estas estrategias de manejo, costo-efectivas, se puede lograr en poblaciones de alto riesgo, de forma racional y similar a como se está trabajando con el cáncer colorrectal (10).

\section{OBJETIVO GENERAL}

Colombia no tiene políticas definidas para controlar y prevenir el CG. Por lo anterior, se establece el siguiente objetivo: con el conocimiento de la historia natural, revisar las estrategias de intervención dirigidas a la prevención primaria y secundaria que permitan disminuir su incidencia y mejorar las cifras de mortalidad y de detección temprana.

\section{HISTORIA NATURAL}

El entendimiento de la historia natural de un tipo de cáncer es crucial en el diseño de una intervención efectiva (11).

En 1975 Pelayo Correa publicó "Un modelo para el desarrollo del cáncer gástrico”. En este artículo argumentó que el desarrollo del CG de tipo intestinal, el subtipo más común, se originaba de un proceso de 30 a 50 años de duración que comenzaba con la gastritis crónica atrófica y progresaba variablemente a metaplasia intestinal, displasia y cáncer. Se postulaba que los cambios iniciales se producían en la primera década de la vida, cuando ocurría la colonización por el H. pylori. Correa inicialmente postuló que los agentes responsables de promover este proceso lento de gastritis a cáncer estaban relacionados con el medio ambiente, basado en los estudios de personas que migraban de áreas de alto riesgo para CG a zonas de bajo riesgo (12).

En su historia natural presenta 3 fases: carcinogénica, asintomática y clínica o sintomática (Tabla 1) (11).

Tabla 1. Fases del CG

\begin{tabular}{ll}
\hline & Historia natural del CG \\
\hline Fase carcinogénica \\
Fase asintomática \\
Fase sintomática
\end{tabular}

\section{Fase carcinogénica (duración de años y décadas)}

\section{Gastritis por H. pylori}

La hipótesis más aceptada propone a la gastritis crónica por $H$. pylori como el factor etiológico inicial del CG. Esta infección se adquiere en la infancia. La lenta y variable progresión a atrofia gástrica, metaplasia intestinal, displasia y adenocarcinoma invasor de tipo intestinal es modulada a lo largo de muchos años por factores genéticos, dietéticos y ambientales, lo que ofrece una amplia oportunidad de intervención $(11,12)$.

Bedoya reportó en 2002 que el $88 \%$ de los niños menores de 10 años mostraba algún cambio inflamatorio en la mucosa gástrica y cambios de gastritis crónica atrófica en el $5 \%(13)$.

Archila y colaboradores en una revisión de biopsias gástricas en población de 1 a 16 años encontraron infección por $H$. pylori en el $59 \%$ de pacientes; en escasa cantidad en el $24,3 \%$, en moderada cantidad en el $20,1 \%$ y en abundante cantidad en el 14,6\% (13).

El H. pylori es el agente causal más importante involucrado en la génesis del CG. La International Agency for Research on Cancer (IARC) lo consideró un agente carcinogénico tipo I desde 1994. El $\mathrm{H}$. pylori está fuertemente asociado con el CG distal del estómago, aunque no muestra ninguna relación con el CG de la región proximal o cardial. Se ha estimado que más del $75 \%$ de los canceres gástricos en todo el mundo se explica por la infección de H. pylori. También existe evidencia de que la infección por el $H$. pylori es una condición necesaria, pero no suficiente para explicar la carcinogénesis gástrica (15).

Otros factores etiológicos como el cigarrillo, la ingesta de alcohol y la formación endógena de nitrosaminas, reconocidos por la IARC, pueden estar involucrados en la causalidad del CG. Entre el $11 \%$ y el $18 \%$ de los casos pueden estar asociados con el cigarrillo. La dieta y la nutrición pueden jugar un papel en la oncogénesis gástrica. Existe evidencia consistente en el que la ingesta de frutas y vegetales están asociados con una disminución en el riesgo para esta enfermedad (Figura 1) (15).

El CG está asociado con una historia familiar (componente genético) con un Odds ratio (OR) de 2 a 8, dependiendo del país. También existen estudios de mayor prevalencia de infección por $H$. pylori y lesiones premalignas en los parientes en primer grado de pacientes con CG, en comparación con los controles $(9,16)$.

La susceptibilidad genética está asumida o relacionada con el CG de tipo difuso causado por una mutación en la E-cadherina, una molécula crucial en el mantenimiento de la arquitectura epitelial (15).

El primer evento crucial en la carcinogénesis gástrica es la infección por el $H$. pylori, lo que desencadena una activación de la respuesta inflamatoria (15). Sin embargo, a pesar de la alta prevalencia de la infección por $H$. pylori, solamente una muy pequeña proporción de pacientes infectados desarrollan CG. De 100 pacientes infectados, solo 1 desarrollará CG (17). 


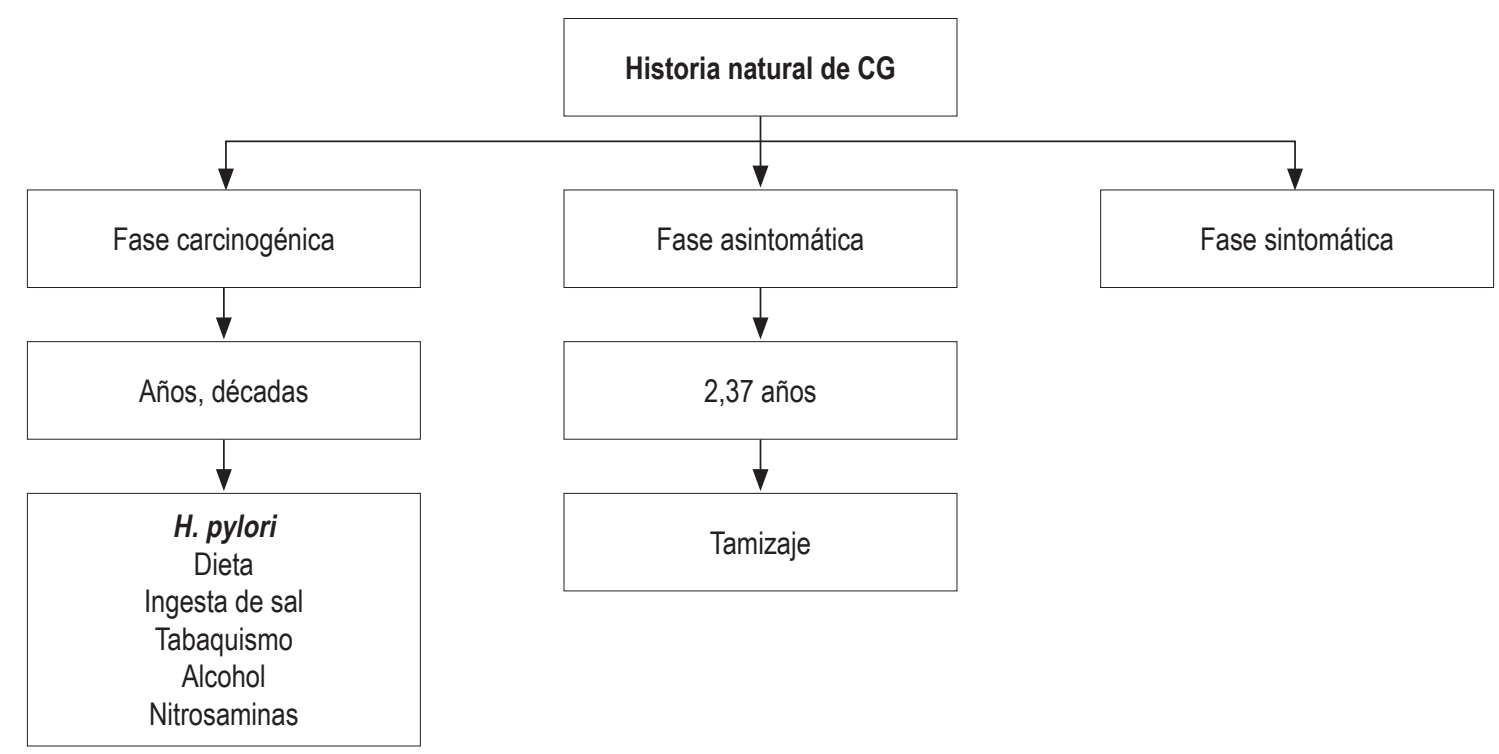

Figura 1. Factores de riesgo para CG. Tomado de: Park JY et al. Clin Endosc. 2014;47(6):478-89.

Esto obliga a preguntarse por qué y cómo la enfermedad se desarrolla en una minoría de pacientes infectados. Una de las razones es la diferencia en la patogenicidad de la bacteria. Las investigaciones en este campo se han enfocado en la susceptibilidad genética (polimorfismos en genes que gobiernan las respuestas de inflamación gástrica), la heterogeneidad del $\mathrm{H}$. pylori y otras influencias del medio ambiente tales como la sal de la dieta o la presencia de especies no Helicobacter dentro de la microbiota gastrointestinal pueden explicar por qué solamente una pequeña proporción de individuos colonizados por el $H$. pylori pueden desarrollar el CG (12).

Considerar al CG como la consecuencia de una infección ha permitido el entusiasmo de diagnosticar y tratar al H. pylori en áreas de alta prevalencia de CG (12). Ha sido claro que la mayoría de los CG se deben a la infección por H. pylori más que a factores relacionados con los estilos de vida. Cuando se sospecha que un cáncer es causado por una infección, se requieren medidas preventivas con el objetivo de disminuir su incidencia y mortalidad (12).

La infección por HP es típicamente adquirida en la infancia y se requieren años y décadas para que exista la transformación de la mucosa por el proceso inflamatorio crónico a estados de gastritis atrófica y metaplasia intestinal. Entonces, la erradicación de la bacteria en individuos jóvenes podría evitar esta progresión y de esta forma disminuir el riesgo de desarrollo del CG más adelante (11).

En Japón en 2009 y en 2103 se estableció, con base en estos argumentos, que la estrategia de diagnosticar y tratar a pacientes con gastritis asociada a $H$. pylori era válido y respaldado por el sistema de seguridad. Esta población, luego del tratamiento erradicador, tendría la mayor proporción de gastritis no atrófica y el seguimiento endoscópico no sería necesario (18).

En la isla de Matsú, en Taiwán, los residentes tienen una alta prevalencia por $H$. pylori. La incidencia de CG es de 50 por 100000 habitantes, la cual es aproximadamente 3 a 5 veces mayor a la incidencia de Taiwán. La edad elegida se estableció a los 30 años con el fin de acelerar la eliminación del CG. Un estudio piloto de tamizaje comenzó en esta población de la isla en 2004 con el objetivo de diagnosticar y tratar la infección por $H$. pylori. Los resultados iniciales fueron muy promisorios. La incidencia de CG disminuyó en un $25 \%$ y la atrofia gástrica en un $77 \%$, en comparación con los datos históricos (19). Se ha calculado que es necesario diagnosticar y tratar a 15 hombres en la China y 245 mujeres en los Estados Unidos para evitar un caso de CG (20).

La prevención de la adquisición de la infección o el tratamiento erradicador del $\mathrm{H}$. pylori antes del desarrollo de la gastritis atrófica son los mejores medios para reducir o eliminar el CG (21).

En 2005, el premio nobel de fisiología fue otorgado a Marshall y Warren por el descubrimiento del H. pylori y su papel en la gastritis y la úlcera péptica. Además, la inflamación crónica es un factor de riesgo común para la carcinogénesis y se sugirió que la prevención primaria del CG podría ser lograda a través de una estrategia de tamizar y tratar la infección del $H$. pylori (11). 
En 2013 un grupo de trabajo de la IARC revisó la evidencia acumulada que soporta el uso de la erradicación masiva del $H$. pylori como una estrategia para prevenir el CG. Con base en los resultados favorables de estudios clínicos controlados y estudios observacionales, un grupo de expertos confirmó que esta estrategia era efectiva (19). Por este motivo, la IARC recomendó a las agencias de cuidado de salud incluir esta estrategia en los programas nacionales de control del cáncer.

En enero de 2014 se realizó un consenso global en Kioto, Japón, para evaluar el manejo de la gastritis crónica asociada con $H$. pylori. De forma similar, en sus conclusiones se estableció que la erradicación del H. pylori podía prevenir el CG y que todos los portadores de H. pylori deberían ser tratados para erradicar este patógeno (21). Si el $\mathrm{H}$. pylori es eliminado de la población, aproximadamente el $75 \%$ de los CG podrían ser eliminados (22).

Nuestra atención debe estar enfocada o dirigida a cómo se realiza esta estrategia, por ejemplo, con la identificación de los pacientes con infección por H. pylori dentro de la población asintomática y la erradicación antes del desarrollo del CG. Sin embargo, la estrategia actual debe depender de la infección por H. pylori y de la incidencia del CG dentro de esa población (23).

En un metaanálisis de 3 estudios (Forman, Parsonnet y Nomura) se encontró un OR de 3,8 de riesgo para desarrollo de CG en personas infectadas por $H$. pylori frente a personas no infectadas. Uemura mostró que los pacientes infectados con $H$. pylori y que no eran tratados tuvieron mayor posibilidad de progresión a CG en los siguientes 12 años, en comparación con los pacientes no infectados (12).

Metaanálisis recientes y estudios de bajo poder estadístico indican que la erradicación del $\mathrm{H}$. pylori disminuye el riesgo del desarrollo de CG en aproximadamente el $40 \%$ en estudios de prevención primaria (individuos asintomáticos) y en un $54 \%$ como estrategia de prevención terciaria (previniendo la aparición de un segundo CG después de la resección endoscópica de un CG temprano). No se sabe si el tratamiento erradicador del $H$. pylori dentro de la cascada de Correa tenga un punto de no retorno $(12,24)$.

En el trabajo de Lee y colaboradores, el cual incluyó 24 publicaciones con más de 48000 individuos con seguimiento de 34000 personas/año (14 estudios de prevención primaria y 10 estudios de prevención terciaria), mostró que el beneficio de la erradicación del $H$. pylori fue más evidente en áreas donde la incidencia de CG es mayor. Sin embargo, la reducción del riesgo fue evidente en casi todo individuo evaluado en el estudio. Es de suponer que las poblaciones de alto riesgo en países de bajo riesgo, incluidos inmigrantes, quienes permanecen infectados desde la infancia, se beneficien de forma importante de la erradicación (12).
544 pacientes con cirugía endoscópica por CG temprano fueron aleatorizados para recibir tratamiento erradicador del H. pylori. El CG metacrónico se detectó en 9 pacientes en el grupo que recibió tratamiento y en 24 pacientes en el grupo que no recibió tratamiento, con una $\mathrm{p}<0,01$. Esto indica que el efecto preventivo de la terapia erradicadora del $H$. pylori en estos pacientes muestra una reducción significativa del riesgo de CG metacrónico (25).

Una de las estrategias de intervención en la historia natural del CG, específicamente en la fase carcinogénica, es la política de diagnosticar y tratar la infección por H. pylori, especialmente antes de que se presente la atrofia gástrica y o la metaplasia intestinal. Pero también la terapia erradicadora debe realizarse en pacientes con atrofia y metaplasia, si está presente la infección por la bacteria. Existirá un momento de no retorno, en el que la terapia no tendrá ninguna justificación porque el daño de la mucosa está hecho (24).

Algunos investigadores están buscando el punto de no retorno. Cuando el $\mathrm{H}$. pylori genera cambios en el ácido desoxirribonucleico (ADN) de la persona, se llega a un punto en que el daño lo lleva a desarrollar cáncer de manera irreversible, aun erradicando la infección (26). Se está determinando cuál es ese punto de no retorno para definir los momentos en los que se recomienda erradicar la infección con el fin de garantizar la recuperación del daño en la mucosa, detener el proceso y prevenir el desarrollo del cáncer.

Un gran número de los profesionales médicos en Colombia no tiene un claro y profundo conocimiento de lo que implica la gastritis en términos de riesgo, historia natural, intervención y seguimiento cuando tiene que enfrentarse a un reporte de patología de gastritis crónica atrófica con o sin metaplasia intestinal (14).

La incidencia de CG se incrementa con la edad, y la magnitud del resultado de la erradicación del $H$. pylori en la incidencia del CG depende de la población estudiada (23).

$\mathrm{La}$ evidencia sugiere que todos los individuos con gastritis por H. pylori deben ser tratados. En países con poblaciones de alto riesgo para CG, esta estrategia es recomendada para individuos jóvenes, menores de 20 años, teniendo en cuenta que la infección es adquirida en la infancia. Este conocimiento puede tener utilidad clínica para estratificar los individuos con infección por $H$. pylori en riesgo alto y riesgo bajo para $\mathrm{CG}$, creando un esquema de seguimiento personalizado (12).

La pregunta sería cómo prevenir el CG (Figura 2). Los resultados apoyan la estrategia de erradicar la bacteria en países donde el $H$. pylori y el CG son comunes. Luego, la estrategia actual debe realizarse dependiendo de la prevalencia del H. pylori y del CG (23). En el consenso de Kioto (2014) los participantes recomendaron de forma unánime la implementación de la terapia de tratamiento erradicador del 


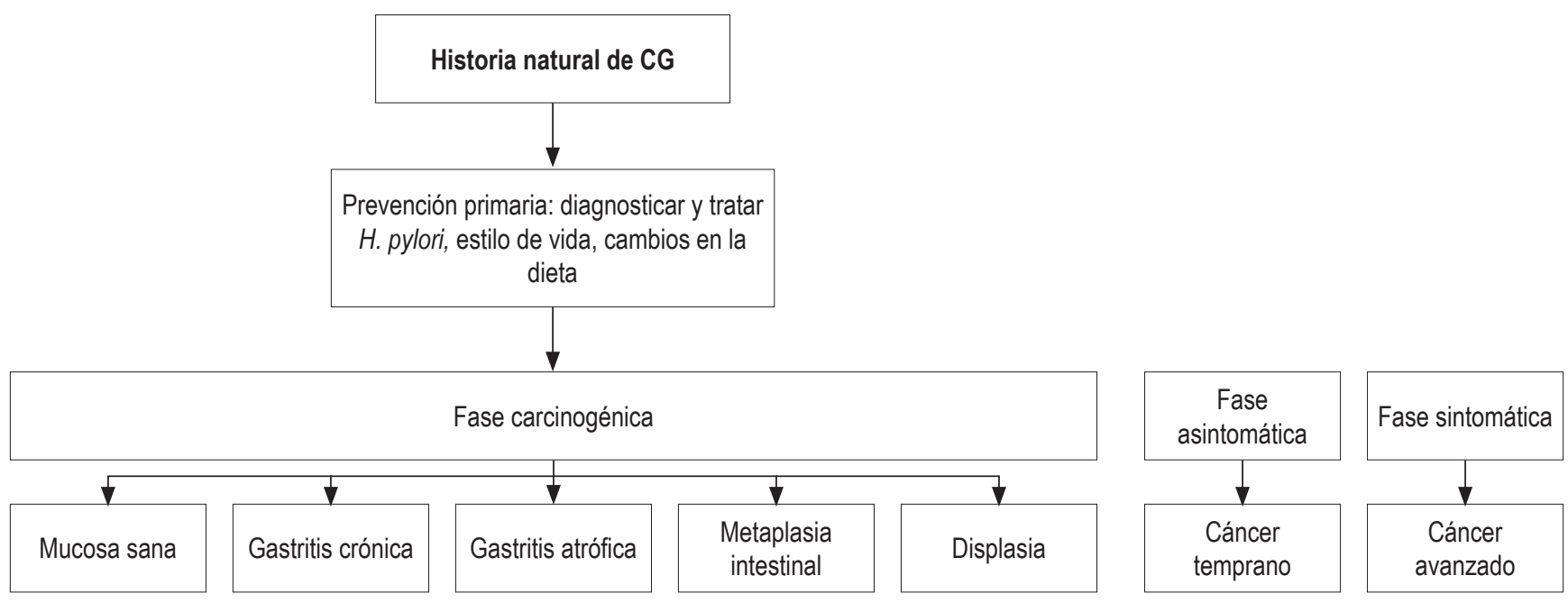

Figura 2. Estrategias de prevención primaria de CG. Tomado de: Lee YC et al. Gut Liver. 2016;10(1):12-26.

H. pylori antes de que los cambios precancerosos se desarrollen (21). La razón: se reduce el riesgo de progresión a atrofia gástrica, metaplasia intestinal y se reduce el riesgo futuro de CG.

Esta estrategia de tamizar y tratar la infección por el $H$. pylori parece ser el mejor abordaje para disminuir el riesgo de cáncer. Sin embargo, la implementación de esta estrategia a nivel de la población requiere un enfoque sistemático. El programa debe ser también integrado dentro de las prioridades del cuidado de salud a nivel nacional, de forma que permita que los recursos limitados sean efectivamente asignados y utilizados. La implementación puede requerir la adopción de una estrategia apropiada. Dentro de la población hay subgrupos que varían en riesgo, de tal forma que es imposible que el abordaje sea el mismo para todos (11).

Tratar todos los pacientes con infección documentada por histología o prueba rápida de ureasa no se justificaría porque no resultaría costo-efectivo. Es necesario definir un grupo de alto riesgo dentro de la población.

Diagnosticar y tratar la infección por $\mathrm{H}$. pylori en individuos asintomáticos procedentes de zonas de alto riesgo para CG (20/100 000 habitantes)

Diagnosticar y tratar la infección por $H$. pylori en pacientes con antecedentes de resección mucosa endoscópica por CG temprano, familiares en primer grado con CG y pacientes sometidos a gastrectomía subtotal $(27,29)$

Figura 3. Resumen de las estrategias de prevención primaria.

\section{Grupo de alto riesgo}

Individuos procedentes de áreas de alto riesgo, especialmente en poblaciones con incidencia mayor de 20/100 000 habitantes, con antecedentes familiares en primer grado con CG, con antecedentes de tabaquismo pesado, ingesta de sal y alcohol (Figura 3) $(24,27)$.

\section{Análisis de costos}

La literatura muestra que los programas de diagnóstico y tratamiento para los pacientes con infección por $H$. pylori es más costo-efectivo en los países donde la incidencia de CG es más alta, en comparación con países de baja incidencia $(30,31)$. Dos estudios demostraron que la edad óptima de tamizaje está entre los 20 a 30 años porque realizarlo en cohortes de mayor edad resultó menos costo-efectivo (32).

La explicación de por qué el tamizaje del $\mathrm{H}$. pylori es costo-efectivo es el resultado del relativo bajo costo de las pruebas y del tratamiento del $H$. pylori y el hecho que el tamizaje se realiza una sola vez. Los costos estimados para la

Diagnosticar y tratar la infección por $H$. pylori en individuos dispépticos sintomáticos con gastritis por $\mathrm{H}$. pylori. Se reduce la progresión a atrofia, metaplasia intestinal y el riesgo futuro de CG (28)

Edad del tratamiento: debe realizarse en la población juvenil (21) 
detección y tratamiento del $H$. pylori fueron menos del $1 \%$ de los costos del tratamiento del CG en todos los estudios. Por tanto, la estrategia de diagnosticar y tratar el $\mathrm{H}$. pylori es una intervención económica con un considerable ahorro en los costos. El consenso de CG recomienda el tamizaje con una prueba serológica. En las poblaciones de alta prevalencia, la serología domina sobre la prueba de aliento; mientras que en las poblaciones de baja prevalencia, la prueba de antígeno fecal domina sobre las otras dos opciones. Debe tenerse en cuenta que la aceptabilidad de la prueba es uno de los requisitos para la introducción de esta estrategia en la población a estudio. Se ha encontrado que la prueba serológica y el antígeno fecal resultaron más costo-efectivos que la prueba de aliento. Repetir el tamizaje o el tratamiento, o limitar el tratamiento a aquellos con cepas CagA no parece ser costo-efectivo (32).

Un estudio a gran escala para la prevención de cáncer de colon y gástrico a través de la detección de sangre oculta en la materia fecal y antígeno fecal para $H$. pylori se está realizando en Taiwán. Los pacientes infectados por H. pylori reciben tratamiento y aún se esperan los resultados de este estudio (11).

\section{Atrofia gástrica}

Debido a que el CG se desarrolla a través de un largo período de años a décadas, la frecuencia de atrofia gástrica es muy baja antes de los 40 años $(<5 \%)$ y el porcentaje de pacientes con CG menores de 40 años corresponde al 5,9\% $(33,34)$.

Un estudio previo de Pelayo Correa realizado en una zona de alta incidencia de CG reportó una prevalencia de gastritis crónica atrófica del $57 \%$, metaplasia intestinal del $38 \%$ y displasia del $10 \%$ en individuos mayores de 40 años (35). Esto significa que la presencia de lesiones precancerosas y su identificación pueden tomar también varios años. Por ello el seguimiento endoscópico en pacientes con alto riesgo puede ayudar a identificar lesiones malignas en estado temprano cuando son todavía operables y tienen alta probabilidad de ser curadas. Un estudio con seguimiento a 10 años reportó que las cifras de progresión a CG para pacientes con gastritis atrófica, metaplasia intestinal, displasia leve y displasia grave son de $0,8 \%, 1,8 \%, 4 \%$ y $33 \%$, respectivamente (36).

Los pacientes con atrofia o metaplasia intestinal extensa deben ser seguidos con endoscopia cada 3 años y los pacientes con atrofia moderada o metaplasia intestinal limitada solo al antro no necesitan seguimiento (37). El manejo debe ser individualizado de acuerdo con otros factores tales como historia familiar de CG, origen geográfico, tabaquismo e ingesta de sal.

La presencia de displasia es un indicador de alto riesgo para CG y debe ser confirmada y clasificada por dos patólogos debido a la variabilidad interobservador.
Las guías europeas han recomendado que los pacientes con gastritis atrófica extensa o metaplasia intestinal extensa deben tener seguimiento endoscópico cada 3 años. La incidencia de CG en 10 años de seguimiento para los pacientes con gastritis atrófica es del $0,8 \%$ y para los pacientes con metaplasia intestinal del $1,8 \%$, por lo que el seguimiento endoscópico de estos 2 grupos debería ser diferente (37).

Se ha establecido un sistema de evaluación de la atrofia (OLGA), basado en el grado de atrofia y su localización. Cuando se está dentro del dominio del diagnóstico y seguimiento de las lesiones premalignas, se recomienda que los pacientes llevados a endoscopia tengan al menos 5 muestras incluidas 2 del antro, 2 del cuerpo y 1 de la incisura. La necesidad de esta última biopsia se basa en la evidencia de que la prevalencia de metaplasia intestinal es mayor en este sitio, en comparación con cualquier otra parte del estómago. La metaplasia intestinal generalmente se inicia en la incisura y se disemina hacia el antro y hacia el cuerpo $(37,38)$.

\section{Metaplasia intestinal}

La metaplasia intestinal se clasifica en completa e incompleta. Si la metaplasia intestinal es reversible o no, es motivo de controversia (39), por lo que se consideraba como un punto de no retorno (40). La metaplasia intestinal completa se considera un proceso reactivo de corto tiempo que generalmente regresa, mientras que la metaplasia intestinal incompleta está relacionada con injurias y cronicidad prolongadas, por lo cual es más factible que progrese a displasia (41).

Los pacientes con metaplasia intestinal pueden tener hasta 10 veces más riesgo de CG que la población general (42). Existe controversia acerca de la utilidad de clasificar la metaplasia intestinal en la práctica clínica. La presencia de metaplasia intestinal incompleta incrementa significativamente el riesgo de CG $(2,42)$, en comparación con la metaplasia intestinal completa $(37,39)$.

La prevención y tratamiento de la atrofia gástrica y la metaplasia intestinal disminuyen la prevalencia del CG. La erradicación de $H$. pylori es el manejo y un paso fundamental. La detección del CG en sus estadios tempranos es la otra estrategia en estos pacientes (40).

Correa propuso un algoritmo para el manejo y seguimiento de las lesiones preneoplásicas. Para pacientes con metaplasia intestinal, debe medirse la presencia de H. pylori y la extensión de la metaplasia intestinal. Si la infección está presente, debe recibir tratamiento; $y$ si la metaplasia intestinal es extensa y es incompleta, la endoscopia digestiva debe repetirse al año y luego cada 3 años si la lesión persiste; de lo contrario no se requiere el seguimiento (43). Por esto, los pacientes con metaplasia intestinal con al menos uno de estos factores de riesgo (metaplasia intestinal incompleta, historia familiar, antecedentes de tabaquismo e ingesta de 
sal) podrían tener mayor riesgo de desarrollar CG y probablemente se beneficiarían de un control y seguimiento endoscópico más intenso y frecuente $(33,37)$.

La endoscopia digestiva tiene mal rendimiento diagnóstico en Occidente, por lo que el diagnóstico de atrofia gástrica y metaplasia intestinal requiere biopsias sistemáticas del cuerpo y del antro (44). El protocolo para estadificación con el sistema OLGA incluye 5 biopsias: 2 del antro, 2 del cuerpo y 1 de la incisura. Un mayor número de biopsias puede aumentar la sensibilidad (37-39).

Un trabajo de casos y controles utilizando el protocolo de OLGA identificó un 61,8 \% más de casos de atrofia en comparación con los protocolos con menos biopsias. Esto podría permitir la corrección del subdiagnóstico de la atrofia gástrica (45).

Por lo anterior se justificaría practicar endoscopia digestiva de calidad a partir de los 40 años en la población de alto riesgo en busca de las lesiones premalignas, que dependiendo de su extensión y del riesgo de CG de acuerdo con el sistema OLGA, definirían la frecuencia de la endoscopia como seguimiento $(7,33,39)$.

La metaplasia intestinal es una condición premaligna que puede resultar de un proceso adaptativo a un estímulo del medio como el de la infección por el $H$. pylori, tabaquismo y alta ingesta de sal (40). En estudios ingleses se evaluaron los beneficios de seguimiento de los pacientes con metaplasia intestinal y se encontró que la incidencia de CG fue del $11 \%$. El seguimiento endoscópico estuvo asociado con la detección más temprana del CG y mejoría en la supervivencia (40).

En estudios europeos de pacientes con metaplasia intestinal, displasia y seguimiento endoscópico, las cifras de detección de cáncer variaron del $33 \%$ al $85 \%$ (40).

En Estados Unidos (poblaciones de bajo riesgo) el riesgo de progresión es bajo y el seguimiento clínico no está indicado a menos que existan otros factores de riesgo para CG como historia familiar o procedencia de países asiáticos o latinoamericanos (42).
Un consenso europeo sugiere que, si la displasia de bajo grado se detecta en un paciente con metaplasia intestinal, debe realizarse un seguimiento endoscópico con mapeo y biopsias dentro de 1 año. La frecuencia ideal de seguimiento endoscópico no es conocida. El seguimiento puede suspenderse cuando 2 endoscopias consecutivas son negativas para displasia. A diferencia de los pacientes con displasia de bajo grado, los de alto grado deben ser llevados a resección quirúrgica o endoscópica, debido a la alta probabilidad de coexistir con adenocarcinoma invasor. El $25 \%$ de los pacientes con displasia de alto grado puede progresar a adenocarcinoma en 1 año. Si se identifica la infección por H. pylori, debe erradicarse (permanece la controversia de si debe realizarse la erradicación empírica cuando se diagnostica la metaplasia intestinal) (42).

La presencia de metaplasia intestinal incompleta es un predictor reconocido de mayor riesgo para el desarrollo de displasia de alto grado o CG en áreas con alta prevalencia como Japón. Varios estudios han concluido que la metaplasia intestinal incompleta identifica pacientes con alto riesgo de desarrollar CG y ellos requieren seguimiento intensivo (Figura 4) (46).

\section{Displasia}

La displasia gástrica es una lesión precancerosa y es el penúltimo estado en la cascada de la oncogénesis gástrica, como lo formula Correa. Por esto la identificación, manejo y seguimiento de esta lesión es importante en la detección temprana y prevención del CG. La displasia usualmente se clasifica como de bajo y alto grado (47).

Los pacientes con displasia generalmente son hombres y 10 años más jóvenes que sus familiares con CG (61,35 años para displasia y 70 años para CG) (47).

La displasia puede encontrarse en cualquier parte del estómago, pero la mayoría de las veces se encuentra en el antro. También la mayoría de las veces la displasia se descubre incidentalmente durante endoscopias de tamizaje (47).
Se propone como estrategia de seguimiento para individuos de alto riesgo de CG la realización de endoscopia a partir de los 40 años y se establece la frecuencia del seguimiento endoscópico $(28,48)$.

Si existe atrofia extensa (antro y cuerpo) y metaplasia intestinal extensa (antro y cuerpo), se debe hacer seguimiento cada 3 años. Estadificación con OLGA $(38,39)$.
La endoscopia convencional de luz blanca requiere de la cromoendoscopia vital y digital junto con la magnificación para una mejor definición de las lesiones premalignas gástricas (39).

Displasia de bajo grado endoscópicamente no definida: seguimiento.

Displasia de bajo grado endoscópicamente definida: resección endoscópica. Displasia de alto grado: resección endoscópica.
La atrofia y la metaplasia intestinal pueden manejarse con dos estrategias: erradicación del $H$. pylori si existe y seguimiento para la detección de CG temprano (40).

El diagnóstico y seguimiento de las lesiones premalignas (atrofia, metaplasia y displasia) es la estrategia más razonable para reducir la incidencia de CG y mejorar el diagnóstico temprano.

Figura 4. Resumen de las estrategias de prevención secundaria. 
El riesgo real de progresión de la displasia a carcinoma no está claro. Es difícil establecer la historia natural de la displasia. Sin embargo, varios estudios han demostrado que la displasia de alto grado tiene un alto riesgo de progresar a carcinoma o cursar con carcinoma sincrónico. Se han reportado cifras que varían del $60 \%$ al $85 \%$ en un intervalo de 4 a 48 meses. También se sabe que el $25 \%$ de los pacientes con displasia de alto grado al cabo de 1 año han progresado a carcinoma $(39,47)$.

En relación con su manejo, las lesiones de alto grado requieren resección endoscópica, debido a su potencial de progresión a carcinoma y a la coexistencia con el carcinoma. Cuando las lesiones no están bien definidas endoscópicamente, se recomienda que se les realice seguimiento 1 año después del diagnóstico. Las lesiones con displasia de alto grado deben manejarse con resección endoscópica (47).

En ocasiones la resección endoscópica está indicada no solo como diagnóstico sino como tratamiento de la displasia.

\section{Fase asintomática (de tamizaje)}

Es el período asintomático durante el cual un cáncer puede detectarse a través de exámenes de tamizaje antes de que los síntomas típicos del diagnóstico aparezcan. Esta fase está definida como el tiempo desde el inicio del cáncer hasta el inicio de los síntomas. Es el momento ideal para los programas de tamizaje (11).

Este período es un concepto teórico, actualmente imposible de medir en cada caso particular, pero es el parámetro estadísticamente más importante para definir el intervalo del tamizaje en la población general (11). Este tiempo se ha definido para el CG de 2,37 años en promedio y es el soporte para el intervalo de tamizaje cada 2 años, como lo han sugerido los coreanos (11). Sin embargo, cambia con la edad del siguiente modo: en la población de 40 a 49 años es 1,25 años; de 50 a 59 años, de 3,18 años; y de 60 a 69 años, de 3,74 años. Esto puede explicar por qué cuando es necesario realizarse el tamizaje con endoscopia en grupos de alto riesgo, este seguimiento debe ser hecho cada año o cada 2 años (11).

Cuando el diagnóstico del cáncer se realiza por tamizaje, la supervivencia puede ser larga y la curación puede ser posible. Sin embargo, el CG tiene un tiempo en esta fase relativamente corto. Los cánceres con fase asintomática larga, como el de próstata, pueden diagnosticarse de forma temprana y asintomática por el beneficio del antígeno prostático específico (PSA), como forma de tamizaje (11).

Por su parte, un CG temprano progresa a CG avanzado en 33 a 48 meses y parte de este tiempo puede cursar asintomático (Figura 5) (49).

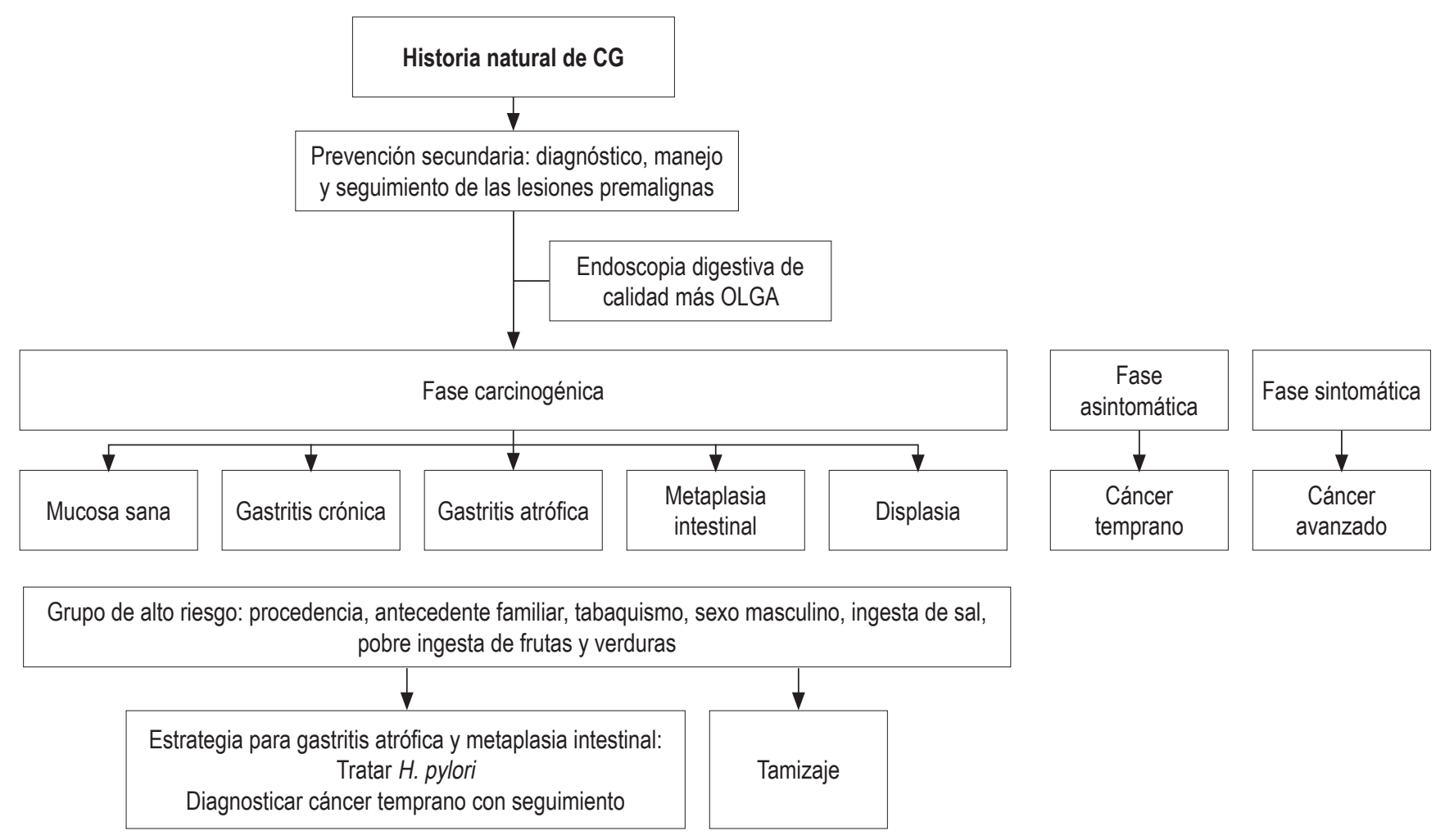

Figura 5. Estrategias de prevención secundaria dentro de la historia natural del CG. 


\section{Fase sintomática}

El CG en su estadio inicial prácticamente es asintomático y cuando aparecen los síntomas la enfermedad se encuentra muy avanzada, momento en el cual el tratamiento quirúrgico curativo frecuentemente es imposible. En esta fase, la supervivencia de los pacientes con CG es de solo el $10 \%$ (50).

El riesgo de desarrollar CG se incrementa con la edad y ocurre más frecuentemente entre los 50 y 80 años de edad. El CG en personas menores de 30 años es raro (6).

En el estudio REGATA, sobre un total de 600 pacientes se encontró que el 5,9\% de ellos era menor de 40 años, 10,1 \% se encontraba entre los 40 y los 49 años, $18,9 \%$ entre los 50 y 59 años y el 65,1 \% era mayor de 60 años. El CG es dos veces más frecuente en los hombres que en las mujeres. En este mismo estudio, el $65 \%$ eran hombres y el $35 \%$ mujeres (34).

Adrada y colaboradores publicaron en 2008 una serie de pacientes con CG y encontró que el 92,4 \% correspondía a lesiones avanzadas (51). Martínez y colaboradores encontraron que el $97 \%$ de los pacientes tenía tumores avanzados (52).

Otro aspecto importante es el costo del manejo de los pacientes con lesiones avanzadas. Gaviria y Cubillos establecieron costos directos (diagnóstico, estadificación, procedimientos médicos y dispositivos médicos) de la atención de los pacientes con CG avanzado, que es de 12 millones para el estadio II y de 27 millones para el estadio III. Se estableció que a mayor estadio de estadificación son mayores los costos (Figura 6) (52).

\section{CONCLUSIONES}

El CG es un candidato ideal para implementar estrategias de prevención. Sin embargo, mientras la prevención primaria se facilita por un objetivo reconocido (H. pylori), las estrategias efectivas de prevención secundaria tienen obstáculos como los altos costos y la necesidad de recursos humanos y técnicos significativos.

Hace más de una década un modelo matemático mostró que el tamizaje de la infección por $H$. pylori y su erradicación podían ser costo-efectivos en países con alta incidencia y mortalidad de CG. También se demostró que el beneficio era solamente significativo en un subgrupo de pacientes sin presencia de lesiones precancerosas. Un metaanálisis de 7 estudios realizados en áreas de alta incidencia de CG demostró una reducción del riesgo de CG entre los pacientes tratados para erradicar el H. pylori (riesgo relativo [RR]:

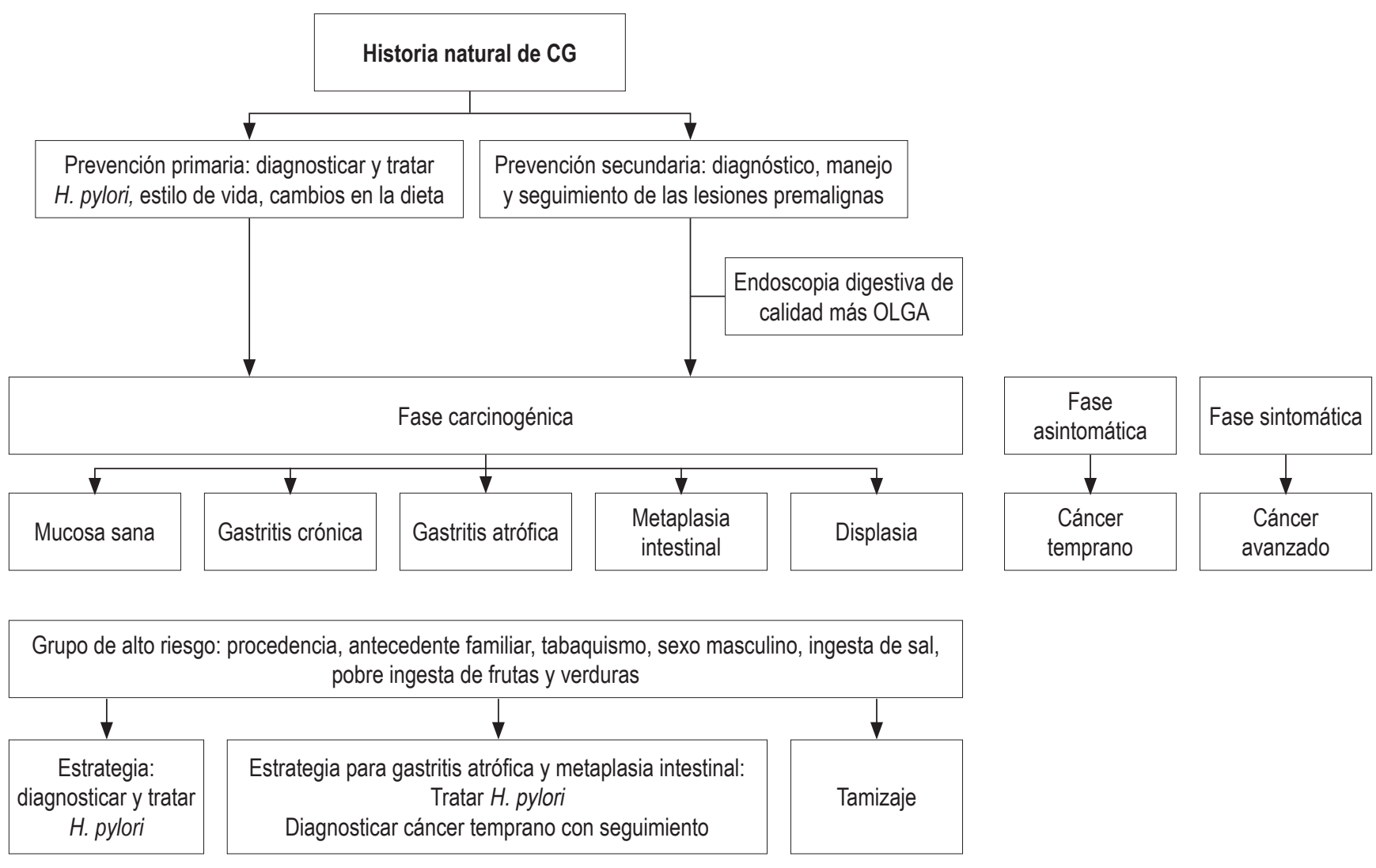

Figura 6. Resumen de las estrategias de prevención primaria y secundaria. Historia natural del CG. 
$0,65)$. Esta estrategia de prevención primaria es costo-efectiva en países con alta incidencia de CG.

El $84 \%$ de los pacientes con CG están por encima de los 50 años, de los cuales el grupo de los 60 a los 70 es el de mayor porcentaje $(65,1 \%)$. Los pacientes con displasia generalmente son hombres y 10 años más jóvenes que sus familiares con CG (61,35 años para displasia y 70 años para CG), por lo que la estrategia para la población promedio debería ser 10 años antes; es decir, se debe iniciar la mayor vigilancia endoscópica y estratificación de riesgo por encima de los 50 años.

La incidencia actual del CG en cualquier población es dependiente de un número de variables: proporción con infección por $H$. pylori, gravedad de la atrofia gástrica y velocidad de desarrollo de la atrofia.

Es necesario cambiar el panorama de esta enfermedad, creando sensibilidad frente a este problema de salud pública dentro del gremio médico y a nivel de los responsables de las políticas de salud. También es necesario el desarrollo de una guía de práctica clínica dirigida a la prevención del CG.

Se deben establecer estrategias de prevención primaria y secundaria que impacten la historia natural del CG.

\section{REFERENCIAS}

1. Den Hoed C, Kuipers E. Gastric Cancer: How can we reduce the incidence of this Disease? Curr Gastroenterol Rep. 2016;18(34):1-8. https://doi.org/10.1007/s11894016-0506-0.

2. Bray F, Ferlay J, Soerjomataram I, Siegel RL, Torre LA, Jemal A. Global cáncer statistics 2018: GLOBOCAN estimates of incidence and mortality worldwide for 36 cancers in 185 countries. CA Cancer J Clin. 2018;68(6):394-424. https:// doi.org/10.3322/caac.21492.

3. ParkJY, von Karsa L, Herrero R. Prevention strategies for gastric cancer: a global perspective. ClinEndosc. 2014;47(6):47889. https://doi.org/10.5946/ce.2014.47.6.478.

4. Crew KD, Neugut AI. Epidemiology of gastric cancer. World J Gastroenterol. 2006;12(3):354-62. https://doi. org/10.3748/wjg.v12.i3.354.

5. World Health Organization. GLOBOCAN 2018: Estimated Cancer Incidence, Mortality and Prevalence Worldwide in 2018. Colombia. WHO [internet] 2018 [acceso 12 de septiembre del 2018]. Disponible en: https://gco.iarc.fr/today/ data/factsheets/populations/170-colombia-fact-sheets.pdf.

6. Piazuelo M, Correa P. Gastric cancer: overview. Colomb Med. 2013;44(3):192-201.

7. Gómez M, Riveros J, Ruiz O, Concha A, Ángel D, Torres M, et al. Guía de práctica clínica para la prevención diagnóstico y tratamiento del cáncer gástrico temprano 2015. Rev Col Gastroenterol. 2015; 30 supl 1:34-42.

8. Correa P. Gastric cancer: overview. Gastroenterol Clin North Am. 2013;42(2):211-7. https://doi.org/10.1016/j. gtc.2013.01.002.
9. Choi IJ. Endoscopic gastric cancer screening and surveillance in high-risk groups. Clin Endosc. 2014;47(6):497503. https://doi.org/10.5946/ce.2014.47.6.497.

10. Binefa G, Rodríguez-Moranta F, Teule A, Medina-Hayas M. Colorectal cancer: from prevention to personalized medicine. World J Gastroenterol. 2014;20(22):6786-808. https://doi.org/10.3748/wjg.v20.i22.6786.

11. Lee YC, Chiang TH, Liou JM, Chen HH, Wu MS, Graham DY. Mass Eradication of Helicobacter pylorito Prevent Gastric Cancer: Theoretical and Practical Considerations. Gut Liver. 2016;10(1):12-26. https://doi.org/10.5009/gnl15091.

12. Moss SF. The Clinical Evidence Linking Helicobacter pylori to GastricCancer.CellMolGastroenterolHepatol.2016;3(2):18391.https://doi.org/10.1016/j.jcmgh.2016.12.001.

13. Archila P, Tovar L, Ruiz M. Características histológicas de la gastritis crónica reportadas en las biopsias gástricas de niños de 1 a 16 años de edad en el Hospital Infantil de San José durante el periodo comprendido entre septiembre de 2008 a septiembre de 2010. Rev Col Gastroenterol. 2012;27(2):74-9.

14. Bedoya A, Sansón F, Yepes Y, Santacruz C, Cifuentes Y, Calvache D, et al. Prevalencia y severidad de las lesiones precursoras de malignidad en un área de alto riesgo de cáncer gástrico. Pasto 2012. Rev Col Gastroenterol. 2012;27(4):275-81.

15. González CA, Agudo A. Carcinogenesis, prevention and early detection of gastric cancer: where we are and where we should go. Int J Cancer. 2012;130(4):745-53. https://doi. org/10.1002/ijc.26430.

16. Yaghoobi M, McNabb-Baltar J, Bijarchi R, Hunt RH. What is the quantitative risk of gastric cancer in the first-degree relatives of patients? A meta-analysis. World J Gastroenterol. 2017;23(13):2435-42. https://doi.org/10.3748/wjg.v23. i13.2435.

17. Yoon H, Kim N. Diagnosis and management of high risk group for gastric cancer. Gut Liver. 2015;9(1):5-17. https:// doi.org/10.5009/gnl14118.

18. Sugimoto $M, B a n H$, Ichikawa $H$, Sahara S, Otsuka T, Inatomi O, et al. Efficacy of the Kyoto Classification of Gastritis in Identifying Patients at High Risk for Gastric Cancer. Intern Med. 2017;56(6):579-86. https://doi.org/10.2169/internalmedicine.56.7775.

19. Leja M, You W, Camargo MC, Saito H. Implementation of gastric cancer screening - the global experience. Best Pract Res Clin Gastroenterol. 2014;28(6):1093-106. https://doi. org/10.1016/j.bpg.2014.09.005.

20. Ford AC, Forman D, Hunt RH, Yuan Y, Moayyedi P. Helicobacter pylori eradication therapy to prevent gastric cancer in healthy asymptomatic infected individuals: systematic review and meta-analysis of randomised controlled trials. BMJ. 2014;348:g3174. https://doi.org/10.1136/bmj.g3174.

21. Sugano K, Tack J, Kuipers EJ, Graham DY, El-Omar EM, Miura S, et al. Kyoto global consensus report on Helicobacter pylori gastritis. Gut. 2015;64(9):1353-67. https://doi. org/10.1136/gutjnl-2015-309252.

22. Wroblewski LE, Peek RM Jr. Helicobacter pylori in gastric carcinogenesis: mechanisms. Gastroenterol Clin North Am. 2013;42(2):285-98. https://doi.org/10.1016/j.gtc.2013.01.006. 
23. Graham DY, Uemura N. Natural history of gastric cancer after Helicobacter pylori eradication in Japan: after endoscopic resection, after treatment of the general population, and naturally. Helicobacter. 2006;11(3):139-43. 10.1111/j.1523-5378.2006.00391.x.

24. Rugge M. Gastric Cancer Risk in Patients with Helicobacter pylori Infection and Following Its Eradication. Gastroenterol Clin North Am. 2015;44(3):609-24. https:// doi.org/10.1016/j.gtc.2015.05.009.

25. Fukase K, Kato M, Kikuchi S, Inoue K, Uemura N, Okamoto $\mathrm{S}$, et al. Effect of eradication of Helicobacter pylori on incidence of metachronous gastric carcinoma after endoscopic resection of early gastric cancer: an open-label, randomised controlled trial. Lancet. 2008;372(9636):392-7. https:// doi.org/10.1016/S0140-6736(08)61159-9.

26. Grisales P. No hay enemigo pequeño: avances contra helicobacter pylori. Pesquisa. 2017;40:14-6.

27. Coelho LG, Maguinilk I, Zaterka S, Parente JM, do Carmo Friche Passos M, Moraes-Filho JP. 3rd Brazilian Consensus on Helicobacter pylori. Arq Gastroenterol. 2013 Apr;50(2). pii: S0004-28032013005000113.https://doi.org/10.1590/ S0004-28032013005000001.

28. Rollán A, Cortés P, Calvo A, Araya R, Bufadel ME, González $\mathrm{R}$, et al. Recommendations of the Chilean Association for Digestive Endoscopy for the management of gastric premalignant lesions. Rev Med Chil. 2014;142(9):1181-92. https://doi.org/10.4067/S0034-98872014000900013.

29. Lee YC, Chiang TH, Chou CK, Tu YK, Liao WC, Wu MS, et al. Association Between Helicobacter pylori Eradication and Gastric Cancer Incidence: A Systematic Review and Meta-analysis. Gastroenterology. 2016;150(5):1113-24.e5. https://doi.org/10.1053/j.gastro.2016.01.028.

30. Asaka M. A new approach for elimination of gastric cancer deaths in Japan. Int J Cancer. 2013;132(6):1272-6. https:// doi.org/10.1002/ijc.27965.

31. Teng AM, Kvizhinadze G, Nair N, McLeod M, Wilson $\mathrm{N}$, Blakely T. A screening program to test and treat for Helicobacter pylori infection: Cost-utility analysis by age, sex and ethnicity. BMC Infect Dis. 2017;17(1):156. https:// doi.org/10.1186/s12879-017-2259-2.

32. Lansdorp-Vogelaar I, Sharp L. Cost-effectiveness of screening and treating Helicobacter pylori for gastric cancer prevention. Best Pract Res Clin Gastroenterol. 2013;27(6):93347. https://doi.org/10.1016/j.bpg.2013.09.005.

33. Rollan A, Ferreccio C, Gederlini A, Serrano C, Torres J, Harris P. Non-invasive diagnosis of gastric mucosal atrophy in an asymptomatic population with high prevalence of gastric cancer. World J Gastroenterol. 2006;12(44):7172-8. https://doi.org/10.3748/wjg.v12.i44.7172.

34. Oliveros R, Navarra LF. Diagnóstico, estadificación y tratamiento del cáncer gástrico en Colombia desde 2004 a 2008 (RegateColombia). Rev Col Gastroenterol. 2012;27(4):269-74.

35. Correa P, Haenszel W, Cuello C, Zabala D, Fontham E, Zarama G, et al. The gastric precursors in a high risk population: cross-sectional studies. Cancer Res. 1990;50:1731-6.
36. den Hoed CM, Holster IL, Capelle LG, de Vries AC, den Hartog B, Ter Borg F, et al. Follow-up of premalignant lesions in patients at risk for progression to gastric cancer. Endoscopy. 2013;45(4):249-56. https://doi.org/10.1055/s-0032-1326379.

37. Zullo A, Hassan C, Repici A, Annibale B. Intestinal metaplasia surveillance: searching for the road-map. World J Gastroenterol. 2013;19(10):1523-6. https://doi. org/10.3748/wjg.v19.i10.1523.

38. Rugge M, Pennelli G, Pilozzi E, Fassan M, Ingravallo G, Russo VM, et al. Gastritis: the histology report. Dig Liver Dis. 2011;43 Suppl 4:S373-84. https://doi.org/10.1016/ S1590-8658(11)60593-8.

39. Dinis-Ribeiro M, Areia M, de Vries AC, Marcos-Pinto R, Monteiro-Soares M, O’Connor A, et al. Management of precancerous conditions and lesions in the stomach (MAPS): guideline from the European Society of Gastrointestinal Endoscopy (ESGE), European Helicobacter Study Group (EHSG), European Society of Pathology (ESP), and the Sociedade Portuguesa de Endoscopia Digestiva (SPED). Endoscopy. 2012;44(1):74-94. https://doi. org/10.1055/s-0031-1291491.

40. Park YH, Kim N. Review of atrophic gastritis and intestinal metaplasia as a premalignant lesion of gastric cancer. J Cancer Prev. 2015;20(1):25-40. https://doi.org/10.15430/ JCP.2015.20.1.25.

41. Lage J, Uedo N, Dinis-Ribeiro M, Yao K. Surveillance of patients with gastric precancerous conditions. Best Pract Res Clin Gastroenterol. 2016;30(6):913-22. https://doi. org/10.1016/j.bpg.2016.09.004.

42. ASGE Standards of Practice Committee, Evans JA, Chandrasekhara V, Chathadi KV, Decker GA, Early DS, et al. The role of endoscopy in the management of premalignant and malignant conditions of the stomach. Gastrointest Endosc. 2015;82(1):1-8. https://doi.org/10.1016/j.gie.2015.03.1967.

43. Correa P, Piazuelo MB, Wilson KT. Pathology of gastric intestinal metaplasia: clinical implications. Am J Gastroenterol. 2010;105(3):493-8. https://doi.org/10.1038/ajg.2009.728.

44. Cañadas R. Metaplasia intestinal gástrica: ¿cómo la estamos abordando? Rev Col Gastroenterol. 2012;27(4):259-62.

45. Martinez D, Otero W, Ricaurte O. Impacto del sistema OLGA en la detección de gastritis crónica atrófica en Colombia: un estudio de casos y controles. Rev Col Gastroenterol. 2016;31(4)360-7.https://doi.org/10.22516/25007440.111.

46. Pittayanon R, Rerknimitr R, Klaikaew N, Sanpavat A, Chaithongrat S, Mahachai $\mathrm{V}$, et al. The risk of gastric cancer in patients with gastric intestinal metaplasia in 5-year followup. Aliment Pharmacol Ther. 2017;46(1):40-45. https:// doi.org/10.1111/apt.14082.

47. Sung JK. Diagnosis and management of gastric dysplasia. Korean J Intern Med. 2016;31(2):201-9. https://doi. org/10.3904/kjim.2016.021.

48. Bisschops R, Areia M, Coron E, Dobru D, Kaskas B, Kuvaev $\mathrm{R}$, et al. Performance measures for upper gastrointestinal endoscopy: A European Society of Gastrointestinal Endoscopy quality improvement initiative. United 
European Gastroenterol J. 2016;4(5):629-656. https://doi. org/10.1177/2050640616664843.

49. Iwai T, Yoshida M, Ono H, Kakushima N, Takizawa K, Tanaka M, et al. Natural History of Early Gastric Cancer: a Case Report and Literature Review. J Gastric Cancer. 2017;17(1):88-92. https://doi.org/10.5230/jgc.2017.17.e9.

50. de Vries E, Uribe C, Pardo C,Lemmens V, Van de PoelE, Forman D. Gastric cancer survival and affiliation to health insurance in a middle-income setting. Cancer Epidemiol. 2015;39(1):91-6. https://doi.org/10.1016/j.canep.2014.10.012.

51. Adrada JC, Calambas F, Díaz JE, Delgado DO, Sierra CH. Características sociodemográficas y clínicas en una pobla- ción con cáncer gástrico en el Cauca, Colombia. Rev Col Gastroenterol. 2008;23(4) 309-14.

52. Martínez J, Garzón M, Lizarazo J, Marulanda JC, Molano JC, Rey M, et al. Características de los pacientes con cáncer gástrico del departamento de Cundinamarca remitidos al Hospital universitario de la Samaritana entre los a-os 20042009. Rev Col Gastroenterol. 2010;25(4): 344-48.

53. Gaviria A, Cubillos L. Costos médicos directos en el tratamiento del cáncer gástrico en los estadios 0 a IIIB en pacientes adultos en Colombia. Colombia: UDCA; 2015. 